\title{
Assistente pessoal para auxiliar na indicação de especialidade médica
}

\author{
Êndril Castilho da Silveira, Rejane Frozza \\ Universidade de Santa Cruz do Sul (UNISC) \\ Departamento de Engenharias, Arquitetura e Computação \\ Curso de Ciência da Computação \\ Av. Independência, 2293 - 96815-900 - Santa Cruz do Sul, RS \\ endrilcs@mx2.unisc.br, frozza@unisc.br
}

\begin{abstract}
Conversational interfaces have gained visibility due to recent technological advances in the use of Artificial Intelligence. In the health area, this type of interface can be very useful for applications that directly interact with the patient, therefore, simulating contact with a health agent. In addition, many patients, when having certain symptoms, usually make a partial self-diagnosis, without a clinical basis, and make an appointment with a specialist doctor, without first asking the opinion of a general practitioner. Consequently, the objective of this research is to propose a chatbot platform in the health area using the Dóris agent, with the functionality of medical pre-consultation to indicate the most appropriate medical specialty for the patient.
\end{abstract}

Resumo. Interfaces conversacionais têm ganhado visibilidade devido aos avanços tecnológicos recentes no uso de Inteligência Artificial. Na área da saúde, esse tipo de interface pode ser útil para aplicações com interação direta com o paciente, simulando, o contato com um agente de saúde. Além disso, muitos pacientes, ao possuírem determinados sintomas, costumam fazer um auto diagnóstico parcial, sem base clínica, e marcam uma consulta com um médico especialista, sem antes pedir a opinião de um clínico geral. Assim, o objetivo desta pesquisa, ainda em andamento, é propor uma plataforma chatbot na área da saúde, utilizando a agente Dóris, com a funcionalidade de pré-consulta médica para indicação da especialidade médica mais adequada ao paciente.

\section{Introdução}

Chatbot é uma aplicação que utiliza entradas e saídas em linguagem natural para realizar uma interação homem-computador, de forma que um diálogo com a máquina seja estabelecido. Nessa interação, o propósito é de emular o comportamento humano. Tal característica fez-se perceber que "antes dos computadores, era possível distinguir pessoas de não-pessoas com base na capacidade de participar de conversas. Mas agora, há modelos híbridos operando entre pessoas e não-pessoas com quem é possível conversar em linguagem comum" (Colby, 1999, p. 6).

Neste contexto de interação, sistemas baseados em conhecimento, como os sistemas especialistas, podem ser utilizados por chatbots para auxiliar na conversa. De 
acordo com Zwass (2016), sistema especialista é um programa de computador que usa métodos de Inteligência Artificial (IA) no intuito de resolver problemas que se baseiam na experiência de um humano. O sistema especialista depende tanto de uma base de conhecimento quanto de um sistema de inferência para simular uma inteligência humana. O sistema de inferência fará o processamento necessário para gerar uma saída, baseado nos dados obtidos na entrada da aplicação e nas informações presentes na base do conhecimento.

Para haver um entendimento na comunicação com seres humanos, as máquinas precisam fazer um processamento da linguagem escrita. Para isso, existem ferramentas, como o IBM Watson, que utilizam a técnica de Processamento de Linguagem Natural (PLN). PLN são técnicas de análise computacional de textos com o propósito de obter o processamento de linguagem semelhante ao processamento humano (Liddy, 2003).

O IBM Watson é um conjunto de serviços de Inteligência Artificial com foco no uso empresarial e comercial. Segundo Shinya (2018), é possível desenvolver agentes de conversação ou chatbots com o Watson Assistant, a Application Programming Interface (API) do Watson para a construção de interfaces conversacionais. A API possui recursos como o PLN e o aprendizado de máquina.

Em um contexto atual de sociedade pós-moderna, tem-se observado uma crescente valorização do tempo de cada pessoa. Além disso, a exponencial evolução tecnológica aliada à automação cada vez mais presente em todos os setores prestadores de serviço, está mudando a percepção da variável tempo no desenvolvimento de atividades e tarefas pelas pessoas. O uso de chatbot neste domínio, inserido na interação entre médico e paciente, pode auxiliar na indicação de uma especialidade médica a partir dos dados coletados pelo diálogo, a fim de otimizar o tempo de encaminhamento de pacientes para as especialidades mais adequadas.

Neste trabalho foi deliberado o seguinte problema de pesquisa: É possível que um chatbot possa auxiliar na indicação de especialidades médicas, a partir das informações coletadas durante a interação com pacientes? O objetivo principal é desenvolver uma plataforma chatbot na área da saúde utilizando a Agente Virtual Conversacional Dóris, que possui interação com usuários via linguagem natural (Cossul et al., 2018). A plataforma terá a funcionalidade de pré-consulta médica para indicação da especialidade médica mais adequada ao paciente.

Juntamente com um médico, especialista humano participante da pesquisa, o sintoma tosse foi escolhido como pré-requisito ao paciente usuário da plataforma. A tosse, ou melhor dizendo, a ação de tossir "é um reflexo natural do aparelho respiratório que surge como consequência de um processo irritativo" (Gargiulo, 2017).

O artigo está organizado nas seguintes seções: a seção 2 descreve a metodologia, na seção 3 são abordados os aspectos referentes à proposta do trabalho e a seção 4 apresenta as considerações finais.

\section{Metodologia}

Até o momento, uma pesquisa exploratória foi realizada, com o levantamento bibliográfico em torno dos assuntos relacionados ao tema e entrevistas com um médico 
generalista, a fim de obter informações técnicas sobre sintomas e patologias variadas. No entanto, a pesquisa será tanto exploratória como descritiva, pois serão analisadas informações coletadas sobre as funcionalidades e interação com o chatbot (Gil, 1991).

Uma bibliometria quantitativa e qualitativa, para levantamento de trabalhos relacionados à pesquisa foi realizada (Araújo, 2006). Na bibliometria quantitativa, buscou-se artigos sem filtro de data de publicação na área de Ciência da Computação, nas bases de dados Web of Science, Scopus e Science Direct. Com os termos de busca em conjunto, "chatbot", "IBM Watson" e "health", não foram encontrados resultados na primeira e apenas 4 e 5 resultados, respectivamente, nas duas últimas. Então, foram selecionados quatro artigos, após leitura dos resumos para serem estudados e contribuírem para o desenvolvimento da pesquisa, sendo eles: Valtolina, Barricelli e Di Gaetano (2019), Denecke et al. (2018), Denecke, Vaaheesan e Arulnathan (2020) e Nadarzynski et al. (2019).

Dentre os artigos analisados, destaca-se o apresentado por Valtolina, Barricelli e Di Gaetano (2019), cujo objetivo foi avaliar os níveis de comunicabilidade entre usuários e sistemas, comparando chatbots e sistemas com interfaces tradicionais nos quesitos de efetividade e eficácia de comunicação, principalmente, com usuários leigos usando essas tecnologias. Os autores sugerem que, para algumas aplicações na área da saúde, uma interface do tipo chatbot pode possuir vantagens em relação às interfaces tradicionais.

No artigo de Denecke, Vaaheesan e Arulnathan (2020) é mencionado que um chatbot pode ter vantagens na interação com humanos, por conta do nível de impessoalidade que a relação com a ferramenta proporciona. $\mathrm{O}$ projeto teve como objetivo principal desenvolver uma aplicação capaz de identificar sentimentos de seus usuários através da troca de mensagens. A aplicação se propõe, também, a auxiliar na manutenção emocional dos usuários, sugerindo exercícios de mindfullness quando julgar necessário.

A partir dos artigos relacionados observou-se que há estudos recentes envolvendo o tema e existe um crescente interesse dos usuários em sistemas que diferem do método tradicional de interface e, dentre eles, chatbot é uma opção interessante. Assim, neste trabalho, é proposto o desenvolvimento de uma plataforma que utiliza uma interface que difere dos modelos tradicionais de interface, aproveitando a tendência de crescimento do interesse em aplicações chatbot.

Os procedimentos metodológicos definidos são: i) pesquisa bibliográfica, com o objetivo de obter aprofundamento nos assuntos envolvidos ao tema de pesquisa; ii) levantamento dos trabalhos relacionados, iniciando pela bibliometria quantitativa, com o intuito de analisar o número de trabalhos existentes nas áreas relacionadas a este trabalho; iii) definição do modelo da plataforma; iv) desenvolvimento da plataforma de conversação interfaceada pela agente Dóris, utilizando o motor de inferência e o Watson Assistant; v) testes de precisão e usabilidade.

\section{Chatbot para indicação de especialidade médica}

A plataforma será desenvolvida como ilustra a Figura 1, com o usuário interagindo com a agente Dóris através de uma interface. Os dados da conversa entre o usuário e a Dóris 
(1) serão enviados para o Watson Assistant através da API da IBM (2), os quais serão processados e interpretados. Então, estas informações serão repassadas ao motor de inferência (3) para ativar o processo de tomada de decisão, que associará as queixas do usuário com a melhor indicação de especialidade médica (4). Este resultado será repassado para a Agente Dóris (5) que apresentará o resultado para o usuário (6).
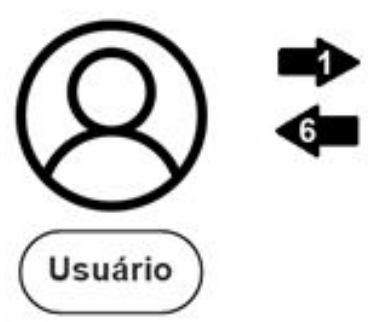
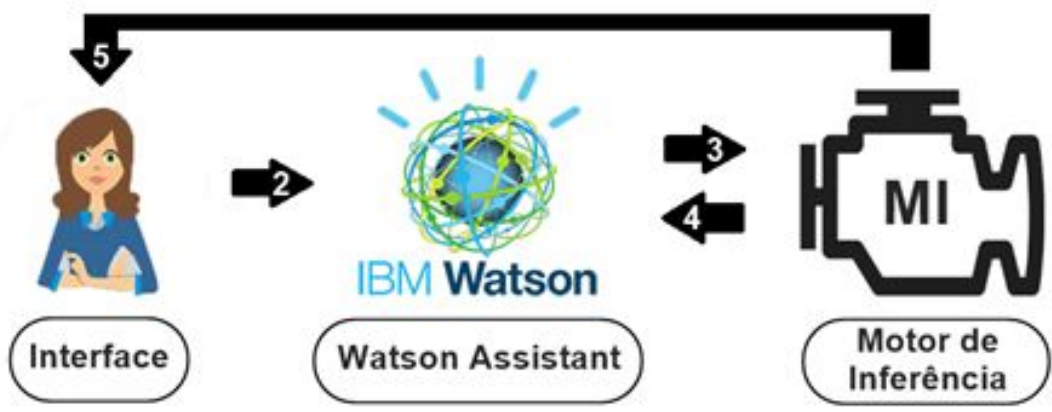

Figura 1. Modelo da plataforma proposta

No momento em que o usuário, neste caso o paciente, iniciar uma conversa, o Watson Assistant identifica por meio da entrada recebida que tipo de assunto o usuário está se referindo. Se identificar uma saudação, por exemplo, a configuração do assistente será suficiente para responder o usuário, então o motor de inferência irá repassar a saída do Watson Assistant diretamente para a interface. No entanto, se o usuário estiver respondendo uma pergunta feita pela plataforma a respeito de seus sintomas, o assistente, após processar a entrada do usuário, receberá uma nova requisição do motor de inferência. Essa nova requisição será utilizada para buscar na base do assistente o próximo questionamento que será feito ao paciente.

O motor de inferência utilizará os sintomas relatados na interação do paciente com a Dóris para inferir a decisão da especialidade médica. Na Figura 2, é possível observar o fluxograma inicial proposto para resolução do problema (diagnóstico). $\mathrm{O}$ motor de inferência será desenvolvido de modo a incorporar este fluxograma em seu funcionamento para o processo de tomada de decisão, ainda que algumas alterações possam se fazer necessárias para melhorar a experiência com o chatbot.

Dentre os métodos de validação propostos para este trabalho, destacam-se dois: i) ainda na fase de testes iniciais da plataforma, perfis de usuários fictícios serão criados com ajuda do especialista humano; o médico irá conferir se a saída fornecida pela plataforma realmente condiz com o esperado; ii) em uma etapa posterior, a plataforma será disponibilizada a um clínico geral que irá convidar seus pacientes a utilizá-la com seu acompanhamento, dessa forma, o especialista humano poderá validar a sugestão que a plataforma apresentar, mas com pacientes reais. 


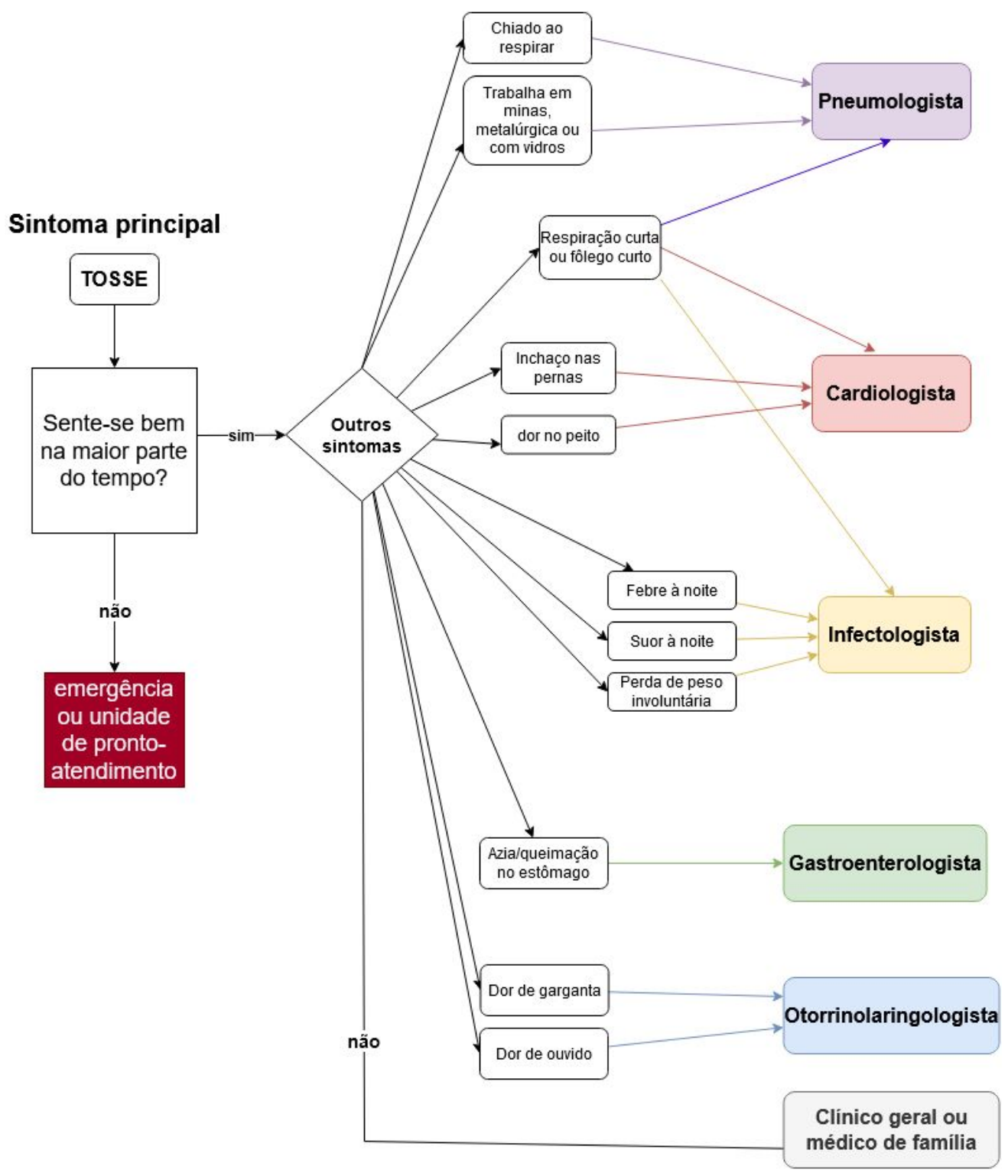

Figura 2. Fluxograma Proposto para Obtenção do Diagnóstico

O fluxograma foi proposto pelo médico especialista humano que participa desta pesquisa e principal ator no processo de aquisição de conhecimento. Este acompanhará o desenvolvimento da plataforma, a fim de garantir que o chatbot possua o melhor desempenho possível em termos de precisão e conduta médica.

\section{Considerações Finais}

A partir dos trabalhos relacionados, verificou-se que as interfaces do tipo chatbot possuem algumas vantagens em comparação às interfaces tradicionais em contextos específicos de aplicações e sua aceitação por parte dos usuários tende a ser positiva. Porém, os trabalhos relacionados normalmente possuem um escopo pequeno, o que abre espaço para novos estudos com abordagens diferentes, no intuito de validação e/ou descoberta científica. Este trabalho pretende ser um destes novos estudos. 
No que se refere à tosse, embora em muitos casos um exame seja indispensável para obter um diagnóstico conclusivo, quando este sintoma possui interações com outros, pode contribuir para o processo de indicação de especialidade médica a ser consultada.

\section{Referências}

ARAÚJO, C. A. Bibliometria: evolução histórica e questões atuais. In: Questão, Porto Alegre, v. 12, n. 1, p. 11-32, 2006.

COLBY, K. M. Comments on human-computer con-versation. In: Wilks, Y. (ed.), Machine Conversations. The Springer International Series in Engineering and Computer Science. Springer, Boston, MA, Vol. 511. 1999.

COSSUL, D.; FROZZA, R.; FAGUNDES, B.J.; FERREIRA, G.; KIPPER, L.M.; WITCZAK, M.V.C. Evolução do agente pedagógico emocional Dóris em um ambiente virtual de aprendizagem. In: Editora Poisson. (Org.). Gestão da Produção em Foco. $1^{\mathrm{a} e}$ ed. Belo Horizonte: Poisson, Vol. 24, p. 28-38. 2018.

DENECKE, K.; HOCHREUTENER, S. L.; POPEL, A.; MAY, R. Self-Anamnesis with a Conversational User Interface: Concept and Usability Study. Methods of Information in Medicine, Vol. 57, No. 5-6, p. 243-252. 2018.

DENECKE, K.; VAAHEESAN S.; ARULNATHAN A. A Mental Health Chatbot for Regulating Emotions (SERMO) - Concept and Usability Test. In: IEEE Transactions on Emerging Topics in Computing, Vol. 1, No. 1, p. 1-1. 2020.

GARGULIO, P. \#Curiosidade: É possível prevenir a gripe utilizando a "Etiqueta da Tosse"? 2017. Disponível em: http://blog.saude.mg.gov.br/2017/06/page/3/. Acessado em maio de 2020.

GIL, A. C. Como elaborar projetos de pesquisa. 3 ed. São Paulo: Atlas, 1991.

LIDDY, E. D. Natural Language Processing. In: Encyclopedia of Library and Information Science. 2a ed. New York: Marcel Decker, 2003, p. 2126-2136

NADARZYNSKI, T.; MILES, O.; COWIE, A.; RIDGE, D. Acceptability of artificial intelligence (AI)-led chatbot services in healthcare: A mixed-methods study. Digital Health, Vol. 5, No. 1, p. 1-12. 2019.

SHINYA, V. Watson Assistant: Como criar o seu chatbot usando Skills e Assistants. 2018. Disponível em: https://medium.com/ibmdeveloperbr/watson-assistant-como-criar-o-seu-chatbot-usando -skills-e-assistants-755b4677984b. Acessado em: maio de 2020.

VALTOLINA, S.; BARRICELLI, B.R.; DI GAETANO, S. Communicability of traditional interfaces VS chatbots in healthcare and smart home domains. In: Behaviour \& Information Technology. Vol. 39, No. 1, p. 108-132. 2019.

ZWASS, V. Expert system. Britannica Academic, Encyclopædia Britannica, 2016. Disponível em: https://www.britannica.com/technology/expert-system. Acessado em: novembro 2019. 